Canadian Science Publishing
nichenem

Canadian Journal of Microbiology Revue canadienne de de microbiologie

\title{
A proteomic approach towards understanding the cross-talk between Bacteroides fragilis and Bifidobacterium longum in co-culture
}

\begin{tabular}{|r|l|}
\hline Journal: & Canadian Journal of Microbiology \\
\hline Manuscript ID & cjm-2015-0804.R2 \\
\hline Danuscript Type: & Note \\
\hline Complete List of Authors: & $\begin{array}{l}\text { Rios-Covian, David; Instituto de Productos Lacteos de Asturias } \\
\text { Sanchez, Borja; Instituto de Productos Lacteos de Asturias } \\
\text { Martinez, Noelia; Instituto de Productos Lacteos de Asturias } \\
\text { Cuesta, Isabel; Instituto de Productos Lacteos de Asturias } \\
\text { Hernandez-Barranco, Ana; Instituto de Productos Lacteos de Asturias } \\
\text { de los Reyes-Gavilan, Clara; Instituto de Productos Lacteos de Asturias } \\
\text { Gueimonde, Miguel; Instituto de Productos Lacteos de Asturias }\end{array}$ \\
\hline \hline Keyword: & Bifidobacterium, Bacteroides, Cross-talk, Interaction, Co-culture \\
\hline & \multicolumn{2}{|l}{} \\
\hline
\end{tabular}

\section{SCHOLARONE \\ Manuscripts}




\section{A proteomic approach towards understanding the cross-talk between}

\section{Bacteroides fragilis and Bifidobacterium longum in co-culture}

4 Authors: David Rios-Covián ${ }^{1}$, Borja Sánchez ${ }^{1}$, Noelia Martínez ${ }^{1}$, Isabel Cuesta ${ }^{2}$, Ana

5 M. Hernández-Barranco ${ }^{2}$, Clara G. de los Reyes-Gavilán ${ }^{1}$ and Miguel Gueimonde ${ }^{1 *}$

71 Probiotics and Prebiotics Group, Department of Microbiology and Biochemistry of 8 Dairy Products, Instituto de Productos Lácteos de Asturias, Consejo Superior de 9 Investigaciones Científicas (IPLA-CSIC), Villaviciosa, Asturias, Spain. Spain.

*Corresponding author: IPLA-CSIC, Paseo Río Linares s/n, 33300 Villaviciosa, Asturias, Spain. Tel.: +34 985892131, Fax: +34 985892233, E-mail: mgueimonde@ipla.csic.es. 


\section{Abstract}

A better understanding of the interactions among intestinal microbes is needed in order to decipher the complex cross-talk that takes place within the human gut. Bacteroides and Bifidobacterium genera are among the most relevant intestinal bacteria and it has been previously reported that co-culture of these two microorganisms affect their survival. Therefore, co-cultures of Bifidobacterium longum NB667 and Bacteriodes fragilis DSMZ2151 were performed with the aim of unravelling the mechanisms involved in their interaction. To this end we applied proteomic (2D-DIGE) analyses and quantified by chromatographic techniques the bacterial metabolites produced during co-incubation. Co-culture stimulated the growth of $B$. longum retarding that of $B$. fragilis, with concomitant changes in the production of some proteins and metabolites of both bacteria. The combined culture promoted up-regulation of the bifidobacterial pyruvate kinase and down-regulation of the Bacteroides phoshoenolpyruvate carboxykinase, two enzymes involved in the catabolism of carbohydrates. Moreover, B. fragilis FKBP-type peptidyl-prolyl cis/trans isomerase, a protein with chaperone-like activity, was found to be over-produced in co-culture, suggesting the induction of a stress response in this microorganism. This study provides mechanistic data to deepen our understanding on the interaction between Bacteroides and Bifidobacterium intestinal populations.

Keywords: Bifidobacterium, Bacteroides, cross-talk, interaction, co-culture 
The intestinal tract constitutes one of the habitats displaying higher bacterial diversity in the human body (Human Microbiome Project Consortium 2012) This complex microbial ecosystem plays an essential role in the maintenance of human health and the disruption of its homeostasis has been linked to several health disorders (Clemente et al. 2012). In spite of its large bacterial diversity, recent studies have indicated that at high phylogenetic level, this ecosystem is dominated by a limited number of bacterial phyla. Among them, Bacteroidetes and Firmicutes are clearly the most abundant in adults, with other phyla such as Actinobacteria, Proteobacteria or Verrumicrobia, being also present at lower levels (Eckburg et al. 2005).

Among Bacteroidetes, the genus Bacteroides represents the most abundant population, constituting about $20-50 \%$ of the colon microbiota (Eckburg et al. 2005). These microorganisms have a predominantly saccharolytic metabolism and are able to ferment host and dietary-derived glucans to succinic, acetic, lactic and propionic acids as the main metabolic end-products (Rios-Covian et al. 2013). The ability of these microorganisms to break-down complex carbohydrates, such as indigestible plant polysaccharides, makes Bacteroides a key player within the intestinal ecosystem (Sonnenburg et al. 2005). Different species of this genus can be present in the intestine, Bacteriodes thetaiotaomicron being the most frequently found in humans (Eckburg et al. 2005). The genus Bacteroides also harbors species that, although often present in the intestinal ecosystem, are important opportunistic pathogens, such as Bacteroides fragilis (Chaudhry and Sharma 2011).

The genus Bifidobacterium is the main representative of the Actinobacteria phylum within the human colon, accounting for up to $3 \%$ of the adult microbiota (Matsuki et al. 2002). Bifidobacteria metabolize sugars by the fructose-6-phosphate phosphoketolase pathway, which leads to the production of acetic and lactic acids as 
main metabolic end-products (Margolles et al. 2011). This genus is among the dominant intestinal bacteria found in the intestine of breast-fed infants, their levels drop later on with weaning and remains stable during the adulthood (Tojo et al. 2014). Diverse species of the genus populate the human gut and Bifidobacterium longum is one of the most abundant. Alterations on the levels and/or species composition of the intestinal bifidobacterial populations have been related with different disease states (Tojo et al. 2014)

Whereas Bacteroides and Bifidobacterium are among the most relevant intestinal microorganisms, the potential interactions between these two bacteria, and of them with complex carbohydrates that may be present in the gut, have attracted the attention of researchers (Adamberg et al. 2014; Falony et al. 2009; Rios-Covian et al. 2013; RiosCovian et al. 2015). In a previous work we observed that the co-culture of $B$. fragilis DSMZ2151 with B. longum NB667 enhanced the survival of the later microorganism (Rios-Covian et al. 2013). In the present study we performed co-culture experiments and proteomic analyses in order to advance a step further into the understanding of such interactions and the potential mechanisms involved.

With this aim, B. longum NB667 (NIZO food research culture collection, EDE, The Netherlands) and B. fragilis DSMZ2151 (DSMZ bacterial collection, Braunschweig, Germany) were incubated in mono-culture and co-culture. Bacterial strains were recovered in MRSc and GAMs broths, respectively, and mono-culture and co-culture experiments were carried out in mCFBM medium supplemented with $0.3 \%$ (w/v) glucose as described previously (Rios-Covian et al. 2013). Mono-cultures were inoculated at $1 \%(\mathrm{v} / \mathrm{v})$ with overnight cultures of B. fragilis or B. longum in mCFBM whereas in co-cultures each of the two strains was inoculated at this percentage. Two samples were collected at T1 $(14 \pm 1$ hours of incubation, mid-late exponential growth 
98 phase, corresponding with $\mathrm{OD}_{600} 0.75 \pm 0.05$ for mono-cultures and $1.05 \pm 0.05$ for the 99 co-culture), and $\mathrm{T} 2(18.5 \pm 1 \mathrm{~h}$ of incubation, early-stationary growth phase, 100 corresponding with $\mathrm{OD}_{600} 1.25 \pm 0.1$ for mono-cultures and co-culture). Bacterial 101 growth (cell counts $\mathrm{mL}^{-1}$ ) was estimated by qPCR using previously described primers 102 and conditions (Arboleya et al. 2012).

103 Cell-free supernatants of mono-cultures and co-cultures were acidified with $\mathrm{HCl}$ 104 to $\mathrm{pH} 2.5 \pm 0.5$ and the SCFA produced during incubation were quantified in the 105 supernatants by Gas Chromatography using a system composed of a 6890 gas 106 chromatograph (Agilent Technologies Inc., Palo Alto, CA, USA) connected with a FID 107 detector (Agilent) as described previously (Salazar et al. 2008). Organic acids and 108 glucose were analyzed by HPLC consisting on an Alliance 2695 separation module and 109 a photo diode array (PDA) detector (Waters 996) at $210 \mathrm{~nm}$ for organic acids, a 110 refractive index detector for glucose (Waters 2414), and Empower software (Waters, 111 Mildford, MA, USA). Chromatographic conditions were those published elsewhere 112 (Salazar et al. 2009). Concentrations are expressed in millimolar (mM).

113 Differences in the proteome of each strain in mono-culture with respect to co114 culture at both $\mathrm{T} 1$ and T2, were assessed separately by two-dimensional difference gel 115 electrophoresis (2D-DIGE) as described previously (Rios-Covian et al. 2015). Mono116 cultures were labelled with Cy3 dye (green color) whereas the co-culture was labelled 117 with Cy5 dye (red color) (Supplementary Fig. 1A). Another gel was performed with a 118 synthetic mixture of the proteins from both mono-cultures labeled with Cy3 and the co119 culture labeled with Cy5 (Supplementary Fig. 1C). This gel was needed in order to 120 detect any new protein spot that may have appeared as a consequence of the co121 cultivation but that was not synthesized by mono-cultures (Supplementary Fig. 1D). No 122 new spots were detected in such conditions. Therefore, we assumed that when 
123 comparing mono-culture $v s$. co-culture (Supplementary Fig. 1A), any spot appearing in

124 the co-culture that was not also present in the corresponding mono-culture (either

125 Bacteroides or Bifidobacterium) belonged to the other microbial partner of the co-

126 culture and, hence, the spot was not considered in the analyses (Supplementary Fig.

127 1B). Gels were analyzed with the 2D Imagemaster (GE Healthcare) software. During

128 the analysis, overlapping spots were excluded. As proteins marked in the co-culture

129 belong to two different microorganisms, an average abundance ratio between proteins in

130 the co-culture relative to the mono-culture was calculated for each strain, representing

131 the average of the abundance ratio obtained for all the spots available in the co-culture

132 for this microorganism. All ratios were normalized by this value and $t$ tests were run

133 between mono-culture and co-culture. Spots displaying a significant change on their

134 production (fold-change higher than 2 or lower than -2) were excised from gels and

135 identified as previously described (Rios-Covian et al. 2015).

136 The relative expression of genes coding for proteins with significant changes in

137 their production on the basis of the 2D-DIGE experiments were further analyzed. RNA

138 was extracted from $10 \mathrm{~mL}$ of cultures taken at the end of the incubation (T2) using the

139 RNeasy mini kit (Qiagen) as reported by Ulve et al. (2008). Gene expression was

140 determined by reverse transcription qPCR (RT-qPCR) using the $\Delta \Delta \mathrm{Ct}$ method as

141 described previously (Gueimonde et al. 2007). Primers used in the present study were: F

142 5'-ATCACTGAACCGACTCCGACA-3' 'and R

143 GCAATGATAAGAATGCAGCACC-3 for $B$. fragilis phosphoenolpyruvate

144 carboxykinase; F 5'-AAAATCTCCTGGTTCAGTTCGC-3' and R 5'-

145 AGGCAACTTCAGCTTCCAGG-3' for B. fragilis FKBP-type peptidyl-prolyl cis-

146 transisomerase; and F 5'-GGATCTCTTCGAGGTTCTCC-3' and R 5'-

147 CACGAGATCATGGACGAGGAA-3' for B. longum pyruvate kinase. The 16S rRNA 
148 gene was employed as endogenous control by using previously described primers 149 (Arboleya et al. 2012) F 5'-GATTCTGGCTCAGGATGAACGC-3' and R 5'150 CTGATAGGACGCGACCCCAT-3' for Bifidobacterium and F 5'151 GagagGaAGGTCCCCCAC-3' and R '5'-CGCKACTTGGCTGGTTCAG-3' for Bacteroides.

One way ANOVA statistical tests were run for the analyses of values obtained

for the different parameters considered between mono-cultures and co-cultures using the IBM SPSS software, version 22.00 (IBM, Armonk, New York, USA).

The results obtained corroborated previous data (Rios-Covian et al. 2013) indicating that co-incubation of B. longum NB667 with B. fragilis DSMZ2151 stimulated the growth of the former microorganism, resulting in significantly higher levels $(\mathrm{p}<0.05)$ of $B$. longum in co-culture than in the corresponding mono-culture $(9.07$ vs. $8.11 \log$ cells $\mathrm{mL}^{-1}$ ) at the end of the incubation (Table 1). In contrast, the growth of B. fragilis was delayed by the presence of the bifidobacteria, as denoted by the significantly lower $(\mathrm{p}<0.05)$ bacterial levels obtained in the co-culture at the first sampling point (T1; representing mild-late exponential phase) (8.66 vs. 8.93 log cells $\mathrm{mL}$ ). This difference, however, disappeared later (T2; early-stationary phase), with $B$. fragilis population reaching similar levels in co-culture than in the corresponding monoculture (8.93 vs. 8.89 log cells $\left.\mathrm{mL}^{-1}\right)$ (Table 1$)$.

As expected from the bacterial growth patterns, the consumption of glucose was higher in the co-culture than in mono-cultures, these differences achieving statistical significance $(\mathrm{p}<0.05)$ at the later sampling point analyzed (Table 1). The enhanced consumption of glucose in co-culture led to higher concentrations of acetate and lactate $(p<0.05)$, which suggests a predominant production by the bifidobacteria. These data are in good agreement with the increased bifidobacterial growth observed for B. longum 
173 when it was grown with B. fragilis. Moreover, in accordance with the delayed growth of

174 B. fragilis in co-culture, the levels of propionate, succinate and formate (mainly

175 produced by Bacteroides with no or very limited production by bifidobacteria) were

176 lower in such conditions than in the corresponding mono-culture (Table 1). These

177 results are in agreement with previous reports (Rios-Covian et al. 2013).

178 To further elucidate the mechanisms involved in this microbe-microbe 179 interaction, the protein pools of B. longum NB667, B. fragilis DSMZ2151 and its co180 culture were analyzed and compared by means of 2D-DIGE. At this point, it is 181 important to underline that the proteomic 2D-DIGE analysis of bacterial co-cultures 182 possess a serious methodological limitation because several spots cannot be analyzed as 183 a consequence of the same gel-migration properties of some proteins of both 184 microorganism, therefore overlapping in the gel (supplementary Fig. 1). In spite of this 185 limitation the proteomic analyses allowed us to identify some spots differentially 186 produced in co-culture with regard to the corresponding mono-cultures. By using as a 187 threshold the occurrence of statistically significant $(\mathrm{p}<0.05)$ changes in production for 188 values greater or lower than two-fold, three spots were found to be affected (Table 2). 189 One of these spots corresponded to the enzyme pyruvate kinase of $B$. longum which was 190 found to be over-produced at both sampling times (T1 and T2). Moreover, RT-qPCR 191 analyses of the expression of the corresponding gene (pyk) at the end of the incubation 192 period (T2) confirmed this observation with more than six-fold induction of the gene 193 expression (Table 2). This enzyme, that catalyzes the conversion of 194 phosphoenolpyruvate to pyruvate, constitutes one of the key steps of sugar metabolism 195 in bifidobacteria (de los Reyes-Gavilán et al. 2005) (Fig. 1). This suggests a stimulation 196 of the catabolism of carbohydrates by B. longum when co-cultured with B. fragilis, thus 197 providing an explanation for its improved growth and the shift on its carbohydrate 
metabolism in co-culture. Interestingly, the pyruvate kinase has been previously found to be over-produced by B. longum (Sanchez et al. 2005; Sanchez et al. 2007) and other microorganisms (Len et al. 2004) under stress conditions. As regarding proteins of $B$. fragilis, the proteomic results evidenced three spots with significant changes in their production in co-cultures with respect to mono-cultures, at least in one of the time points analyzed (Table 2). Two of these spots, likely isoforms, were identified by MALDI TOF/TOF tandem mass spectrometry as a FKBP-type peptidyl-prolyl cis/trans isomerase. This enzyme catalyzes the cis/trans isomerization of peptide bonds adjacent to proline residues (Janowski et al. 1997). In bacteria, this enzyme plays an important role in native protein folding and re-naturalization of denatured proteins and, therefore, it has chaperone-like activity (Jo et al. 2015). Different proteins involved in protein folding and turnover have been found to be up- or down-regulated in B. fragilis when grown in complex carbohydrates, such as bifidobacterial exopolysaccharides (RiosCovian et al. 2015). Moreover, chaperones production in bacteria is often affected by stress factors (Ruiz et al. 2011). In this context, our results suggest that the presence of B. longum in the co-culture promotes a response in B. fragilis leading to a modification on the production of this chaperone. However, the direction of this modification remains unclear as according to the proteomic results there is an up-regulation of the protein production at both sampling times ( $\mathrm{T} 1$ and $\mathrm{T} 2$ ) but, on the contrary, the expression analyses at the end of incubation (T2) indicate a repression of the gene expression (Table 2). To this regard, discrepancies between protein and gene expression analyses are not un-common (Aakko et al. 2014; Wu et al. 2011). It must be pointed out that we employed two different techniques, each with its own performance and limitations. RT-qPCR quantifies the mRNA, whose life-span is relatively short, whilst proteomics quantify the protein accumulated in the cell at a given time, including the 
223 effect of post-translational regulation. These differences may account for the apparently

224 contradictory results obtained.

225 The second $B$. fragilis protein found to be affected by the co-incubation with $B$.

226 longum was the phoshoenolpyruvate carboxykinase, a key enzyme in the catabolism of

227 Bacteroides. This enzyme, catalyzing the conversion of phosphoenolpyruvate to

228 oxalacetate (Figure 1), was found to be under-produced at T1 by proteomics and was

229 found down-regulated at the end of incubation (T2) by the RT-qPCR experiments

230 (Table 2). This down-regulation suggests that co-cultivation with B. longum promotes a

231 shift on the central sugar catabolism of B. fragilis, which may be focused to the use of

232 complex carbohydrates and proteins in order to outcompete B. longum. Supporting this

233 hypothesis are our previous results showing down-regulation of the

234 phosphoenolpyruvate carboxykinase and an activation of the catabolism of amino acids

235 by $B$. fragilis when grown in media containing bacterial exopolysaccharides (complex

236 carbohydrates that promote slow growth) as carbon source with respect to glucose

237 (Rios-Covian et al. 2015). Bacteroides is well adapted to the use of complex

238 polysaccharides and an expansion on its ability to degrade these substrates has been

239 observed when gnotobiotic animals mono-colonized by Bacteroides were compared

240 with those co-colonized by Bacteroides and B. longum (Sonnenburg et al. 2006).

241 Changes observed by us on the Bacteroides metabolism in co-culture with $B$. longum

242 could account for the delayed bacterial growth and reduced metabolites production

243 observed in such conditions.

244 To sum up, the results of this study provide new insights into the physiological

245 and molecular mechanisms that determine the interaction of B. fragilis and B. longum,

246 two important intestinal microorganisms, when growing together. B. fragilis in the co-

247 culture seems to stimulate the growth and metabolic activity of $B$. longum when glucose 
248 is available as a carbon source. The contrary seems to be true for B. fragilis, whose

249 growth was inhibited and the metabolites production reduced in the presence of $B$.

250 longum. The proteomic data allowed identifying the central sugar catabolism pathways

251 as key regulators of this interaction, underlining the role of metabolic interactions

252 among microorganisms as drivers of the cross-talk between these two intestinal

253 bacteria. Increasing our understanding on the microbe-microbe interactions among

254 intestinal bacteria will allow the development of high efficacious and specific

255 microbiota-modulation strategies for improving human health.

\section{ACKNOWLEDGEMENTS}

257 This work was financed by projects AGL2010-16525 and AGL2013-43770-R from

258 Plan Nacional/Plan Estatal de I+D+I (Spanish Ministry of Economy and

259 Competitiveness, MINECO) and the Grant GRUPIN14-043 from "Plan Regional de

260 Investigación del Principado de Asturias”. Both, national and regional grants received

261 cofounding from European Union FEDER funds. David Ríos-Covián was the recipient

262 of a predoctoral FPI fellowship whereas Borja Sánchez enjoys a Ramon \& Cajal

263 contract, both from MINECO.

264 REFERENCES

265 Aakko, J., Sanchez, B., Gueimonde, M., and Salminen, S. 2014. Assessment of stress tolerance 266 acquisition in the heat-tolerant derivative strains of Bifidobacterium animalis subsp. lactis 267 BB-12 and Lactobacillus rhamnosus GG. J. Appl. Microbiol. 117(1): 239-248.

268 Adamberg, S., Tomson, K., Vija, H., Puurand, M., Kabanova, N., Visnapuu, T., Jõgi, E., 269 Alamäe, T., and Adamberg, K. 2014. Degradation of fructans and production of propionic 270 acid by Bacteroides thetaiotaomicron are enhanced by shortage of amino acids. Front. $271 \quad$ Nutr. 1: 00021.

272 Arboleya, S., Binetti, A., Salazar, N., Fernandez, N., Solis, G., Hernandez-Barranco, A., 273 Margolles, A., de Los Reyes-Gavilan, C.G., and Gueimonde, M. 2012. Establishment and 274 development of intestinal microbiota in preterm neonates. FEMS Microbiol. Ecol. 79(3): $275 \quad 763-772$. 
Clemente, J.C., Ursell, L.K., Parfrey, L.W., and Knight, R. 2012. The impact of the gut microbiota on human health: an integrative view. Cell 148(6): 1258-1270.

Chaudhry, R., and Sharma, N. 2011. Bacteroides. In Molecular Detection of Human Bacterial Pathogens. Liu D. (ed.). CRC Press. pp. 491-500.

De Los Reyes-Gavilán, C.G., Ruas-Madiedo, P., Noriega, L., Cuevas, I., Sánchez, B., and Margolles, A. 2005. Effect of acquired resistance to bile salts on enzymatic activities involved in the utilisation of carbohydrates by bifidobacteria. An overview. Lait. 85(1-2): 113-123.

Eckburg, P.B., Bik, E.M., Bernstein, C.N., Purdom, E., Dethlefsen, L., Sargent, M., Gill, S.R., Nelson, K.E., and Relman, D.A. 2005. Diversity of the human intestinal microbial flora. Science. 308(5728): 1635-1638.

Falony, G., Calmeyn, T., Leroy, F., and De Vuyst, L. 2009. Co-culture fermentations of Bifidobacterium species and Bacteroides thetaiotaomicron reveal a mechanistic insight into the prebiotic effect of inulin-type fructans. Appl. Environ. Microbiol. 75(8): 2312-2319.

Gueimonde, M., Noriega, L., Margolles, A., and de los Reyes-Gavilan, C.G. 2007. Induction of alpha-L-arabinofuranosidase activity by monomeric carbohydrates in Bifidobacterium longum and ubiquity of encoding genes. Arch. Microbiol. 187(2): 145-153.

Human Microbiome Project Consortium. 2012. Structure, function and diversity of the healthy human microbiome. Nature. 486(7402): 207-214.

Janowski, B., Wollner, S., Schutkowski, M., and Fischer, G. 1997. A protease-free assay for peptidyl prolyl cis/trans isomerases using standard peptide substrates. Analytical biochemistry 252(2): 299-307.

Jo, G.A., Lee, J.M., No, G., Kang, D.S., Kim, S.H., Ahn, S.H., and Kong, I.S. 2015. Isolation and characterization of a $17-\mathrm{kDa}$ FKBP-type peptidyl-prolyl cis/trans isomerase from Vibrio anguillarum. Prot. Express. Purif. 110: 130-137.

Len, A.C., Harty, D.W., and Jacques, N.A. 2004. Proteome analysis of Streptococcus mutans metabolic phenotype during acid tolerance. Microbiology 150(Pt 5): 1353-136

Margolles, A., Ruas-Madiedo, P., de los Reyes-Gavilán, C.G., Sánchez, B., and Gueimonde, M. 2011. Bifidobacterium. In Molecular Detection of Human Bacterial Pathogens. Liu D. (ed.). CRC Press. pp. 45-57.

Matsuki, T., Watanabe, K., Fujimoto, J., Miyamoto, Y., Takada, T., Matsumoto, K., Oyaizu, H., and Tanaka, R. 2002. Development of 16S rRNA-gene-targeted group-specific primers for the detection and identification of predominant bacteria in human feces. Appl. Environ. Microbiol. 68(11): 5445-5451.

Rios-Covian, D., Arboleya, S., Hernandez-Barranco, A.M., Alvarez-Buylla, J.R., RuasMadiedo, P., Gueimonde, M., and de los Reyes-Gavilan, C.G. 2013. Interactions between Bifidobacterium and Bacteroides species in cofermentations are affected by carbon 
sources, including exopolysaccharides produced by bifidobacteria. Appl. Environ. Microbiol. 79(23): 7518-7524.

Rios-Covian, D., Sanchez, B., Salazar, N., Martinez, N., Redruello, B., Gueimonde, M., and de Los Reyes-Gavilan, C.G. 2015. Different metabolic features of Bacteroides fragilis growing in the presence of glucose and exopolysaccharides of bifidobacteria. Front. Microbiol. 6: 825.

Ruiz, L., Ruas-Madiedo, P., Gueimonde, M., de Los Reyes-Gavilan, C.G., Margolles, A., and Sanchez, B. 2011. How do bifidobacteria counteract environmental challenges? Mechanisms involved and physiological consequences. Genes Nutr. 6(3): 307-318.

Salazar, N., Gueimonde, M., Hernandez-Barranco, A.M., Ruas-Madiedo, P., and de los ReyesGavilan, C.G. 2008. Exopolysaccharides produced by intestinal Bifidobacterium strains act as fermentable substrates for human intestinal bacteria. Appl. Environ. Microbiol. 74(15): 4737-4745.

Salazar, N., Prieto, A., Leal, J.A., Mayo, B., Bada-Gancedo, J.C., de los Reyes-Gavilan, C.G., and Ruas-Madiedo, P. 2009. Production of exopolysaccharides by Lactobacillus and Bifidobacterium strains of human origin, and metabolic activity of the producing bacteria in milk. J. Dairy Sci. 92(9): 4158-4168.

Sanchez, B., Champomier-Verges, M.C., Anglade, P., Baraige, F., de Los Reyes-Gavilan, C.G., Margolles, A., and Zagorec, M. 2005. Proteomic analysis of global changes in protein expression during bile salt exposure of Bifidobacterium longum NCIMB 8809. J. Bacteriol. 187(16): 5799-5808.

Sanchez, B., Champomier-Verges, M.C., Collado, M.C., Anglade, P., Baraige, F., Sanz, Y., de los Reyes-Gavilan, C.G., Margolles, A., and Zagorec, M. 2007. Low-pH adaptation and the acid tolerance response of Bifidobacterium longum biotype longum. Appl. Environ. Microbiol. 73(20): 6450-6459.

Sonnenburg, J.L., Xu, J., Leip, D.D., Chen, C.H., Westover, B.P., Weatherford, J., Buhler, J.D., and Gordon, J.I. 2005. Glycan foraging in vivo by an intestine-adapted bacterial symbiont. Science. 307(5717): 1955-1959.

Sonnenburg, J.L., Chen, C.T., and Gordon, J.I. 2006. Genomic and metabolic studies of the impact of probiotics on a model gut symbiont and host. PLoS Biol. 4(12): e413.

Tojo, R., Suarez, A., Clemente, M.G., de los Reyes-Gavilan, C.G., Margolles, A., Gueimonde, M., and Ruas-Madiedo, P. 2014. Intestinal microbiota in health and disease: role of bifidobacteria in gut homeostasis. World J. Gastroenterol. 20(41): 15163-15176.

Ulve, V.M., Monnet, C., Valence, F., Fauquant, J., Falentin, H., and Lortal, S. 2008. RNA extraction from cheese for analysis of in situ gene expression of Lactococcus lactis. J. Appl. Microbiol. 105(5): 1327-1333. 
349 Wu, R., Zhang, W., Sun, T., Wu, J., Yue, X., Meng, H., and Zhang, H. 2011. Proteomic analysis of responses of a new probiotic bacterium Lactobacillus casei Zhang to low acid stress. Int.

351 J. Food Microbiol. 147; 181-187.

352

353

354

355 
357 Table 1.Glucose consumption, SCFA and organic acid concentrations $(\mathrm{mM})$ and bacterial counts $\left(\log\right.$ cells $\left.\mathrm{mL}^{-1}\right)$ in co-cultures 358 of B. fragilis DSMZ2151 and B. longum NB667 at mid-late exponential (T1) and early-stationary (T2) phases of growth.

\begin{tabular}{|c|c|c|c|c|c|c|c|c|c|}
\hline \multirow{2}{*}{ Time } & \multirow{2}{*}{ Culture } & \multirow{2}{*}{$\begin{array}{c}\text { Glucose } \\
\text { consumption }\end{array}$} & \multirow{2}{*}{ Acetate } & \multirow{2}{*}{ Propionate } & \multirow{2}{*}{ Lactate } & \multirow{2}{*}{ Succinate } & \multirow{2}{*}{ Formate } & \multicolumn{2}{|c|}{ Bacterial counts } \\
\hline & & & & & & & & Bacteroides & Bifidobacterium \\
\hline \multirow[t]{3}{*}{$\mathrm{T} 1$} & DSM2151 & $0.91 \pm 0.06^{\mathrm{a}}$ & $3.24 \pm 0.01^{\mathrm{a}}$ & $0.82 \pm 0.01^{\mathrm{b}}$ & $0.77 \pm 0.04^{\mathrm{a}}$ & $0.92 \pm 0.03^{\mathrm{c}}$ & $2.51 \pm 0.10^{\mathrm{b}}$ & $8.93 \pm 0.16^{*}$ & - \\
\hline & NB667 & $3.35 \pm 1.18^{\mathrm{b}}$ & $8.91 \pm 1.02^{\mathrm{b}}$ & - & $3.46 \pm 0.37^{\mathrm{c}}$ & $0.20 \pm 0.03^{\mathrm{a}}$ & $0.97 \pm 0.13^{\mathrm{a}}$ & - & $8.59 \pm 0.77$ \\
\hline & Co-culture & $3.58 \pm 0.41^{\mathrm{b}}$ & $7.99 \pm 0.07^{\mathrm{b}}$ & $0.61 \pm 0.01^{\mathrm{a}}$ & $2.77 \pm 0.10^{\mathrm{b}}$ & $0.80 \pm 0.02^{\mathrm{b}}$ & $2.20 \pm 0.06^{\mathrm{b}}$ & $8.66 \pm 0.09 *$ & $8.79 \pm 0.19$ \\
\hline \multirow[t]{3}{*}{$\mathrm{T} 2$} & DSM2151 & $1.86 \pm 0.30^{\mathrm{a}}$ & $4.06 \pm 0.09^{\mathrm{a}}$ & $1.08 \pm 0.06^{\mathrm{b}}$ & $4.59 \pm 0.30^{\mathrm{a}}$ & $0.86 \pm 0.16^{\mathrm{b}}$ & $2.81 \pm 0.06^{\mathrm{c}}$ & $8.93 \pm 0.09$ & - \\
\hline & NB667 & $8.70 \pm 0.13^{b}$ & $16.70 \pm 0.34^{\mathrm{b}}$ & - & $8.46 \pm 0.23^{\mathrm{b}}$ & $0.21 \pm 0.01^{\mathrm{a}}$ & $0.57 \pm 0.03^{\mathrm{a}}$ & - & $8.11 \pm 0.32 *$ \\
\hline & Co-culture & $10.26 \pm 0.03^{\mathrm{c}}$ & $18.39 \pm 0.26^{\mathrm{c}}$ & $0.32 \pm 0.01^{\mathrm{a}}$ & $10.51 \pm 0.17^{\mathrm{c}}$ & $0.66 \pm 0.02^{\mathrm{b}}$ & $0.93 \pm 0.05^{\mathrm{b}}$ & $8.89 \pm 0.41$ & $9.07 \pm 0.29 *$ \\
\hline
\end{tabular}

Different letter within the same-time point indicate significant differences among cultures. Asterisks indicate significant differences between mono-culture and co-culture. - not determined/not detected (below detection limit) 
Table 2. Identification of proteins affected by co-cultivation of B. fragilis DSMZ2151 and B. longum NB667 and expression of the corresponding genes

\begin{tabular}{|c|c|c|c|c|c|c|c|c|c|c|c|}
\hline \multirow{2}{*}{ COG orthology } & \multirow{2}{*}{ Spot no. ${ }^{\text {a }}$} & \multirow{2}{*}{ Putative function ${ }^{\mathrm{b}}$} & \multirow{2}{*}{ GI number ${ }^{\mathrm{c}}$} & \multirow{2}{*}{$\operatorname{Mass}^{\mathrm{d}}$} & \multirow{2}{*}{$\mathrm{pI}^{\mathrm{d}}$} & \multirow{2}{*}{$\begin{array}{l}\text { MASCOT } \\
\text { score }\end{array}$} & \multirow{2}{*}{$\begin{array}{l}\text { No. of } \\
\text { peptides } \\
\text { matched }^{\text {e }}\end{array}$} & \multirow{2}{*}{ Coverage } & \multicolumn{2}{|c|}{ Protein change fold ${ }^{\mathrm{f}}$} & $\begin{array}{l}\text { Gene expression change } \\
\text { fold }^{\mathrm{g}}\end{array}$ \\
\hline & & & & & & & & & $\mathrm{T} 1$ & $\mathrm{~T} 2$ & $\mathrm{~T} 2$ \\
\hline $\begin{array}{l}\text { Energy production } \\
\text { and conversion }\end{array}$ & Bac25 & phosphoenolpyruvate carboxykinase & gi|53715725 & 59333 & 5.73 & 333 & 15 & 25 & -2.15 & ND & $-3.06 \pm 1.47$ \\
\hline $\begin{array}{l}\text { Posttranslational } \\
\text { modification, protein } \\
\text { turnover, chaperones }\end{array}$ & Bac44 & $\begin{array}{l}\text { FKBP-type peptidyl-prolyl cis- } \\
\text { transisomerase }\end{array}$ & gi|547946109 & 51380 & 4.69 & 155 & 11 & 19 & 2.99 & ND & $-2.84 \pm 0.80$ \\
\hline \multirow{3}{*}{$\begin{array}{c}\text { Carbohydrate } \\
\text { transport and } \\
\text { metabolism }\end{array}$} & Bif8 & Pyruvate kinase & gi|312133003 & 52310 & 5.24 & 525 & 29 & 49 & ND & 2.95 & \multirow{3}{*}{$6.55 \pm 1.25$} \\
\hline & Bif9 & Pyruvate kinase & gi|312133003 & 52310 & 5.24 & 587 & 34 & 54 & ND & 4.54 & \\
\hline & Bif10 & Pyruvate kinase & gi|312133003 & 52310 & 5.24 & 527 & 33 & 49 & 2.22 & 2.17 & \\
\hline
\end{tabular}

${ }^{\mathrm{a}}$ Spot numbers refer to the proteins labelled in 2D-DIGE gels. B. fragilis, Bac; B. longum, Bif.

${ }^{b}$ Putative functions were assigned from the NCBI gene database

${ }^{\mathrm{C}} \mathrm{GI}$ number in the NCBInr database for B. fragilis DSMZ2141 and B. longum NB667

${ }^{\mathrm{d}}$ As given by the NCBInr databse for B. fragilis DSMZ2151 and B. longum NB667. Molecular masses are expressed in kilodaltons

${ }^{\mathrm{e}}$ Number of tryptic peptides observed that contributed to the percentage of amino acid coverage.

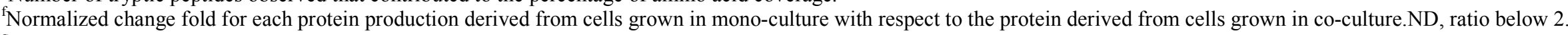

${ }^{\mathrm{g}} \mathrm{Gene}$ expression change fold for each gen derived from cDNA obtained from cells grown in mono-culture with respect to the cDNA obtained from cells grown in co-culture.16s

RNA gene was used as housekeeping gene. 


\section{FIGURE LEGENDS}

379 Figure 1.Schematic representation of $B$. fragilis and B. longum metabolism. Final 380 metabolites are rounded. Dark arrows indicate one reaction step, dashed arrows indicate 381 more than one reaction. Phospoenolpyruvate, PEP; Oxalacetate, OAA.1, pyruvate 382 kinase; 2, phospoenolpyruvate carboxykinase.

383 

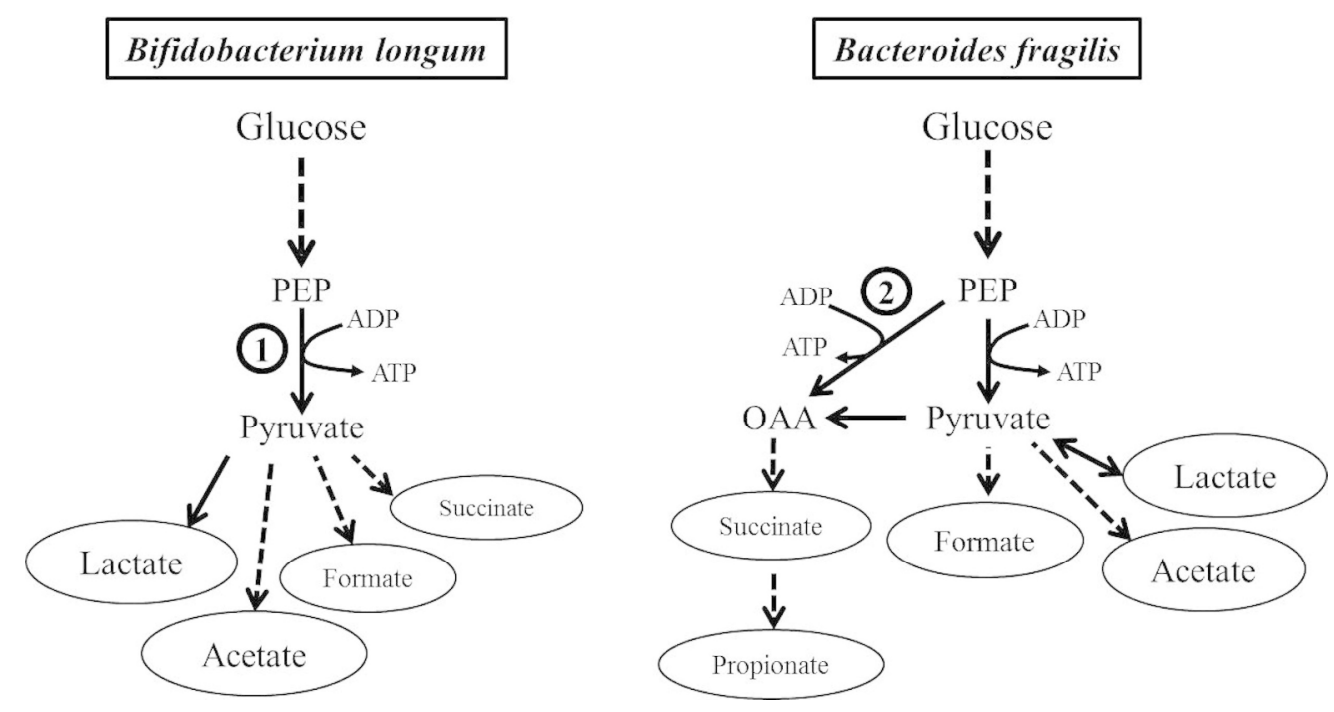

Figure 1.Schematic representation of B. fragilis and B. Iongum metabolism. Final metabolites are rounded. Dark arrows indicate one reaction step, dashed arrows indicate more than one reaction. Phospoenolpyruvate, PEP; Oxalacetate, OAA.1, pyruvate kinase; 2, phospoenolpyruvate carboxykinase. $317 \times 166 \mathrm{~mm}(200 \times 200$ DPI $)$ 